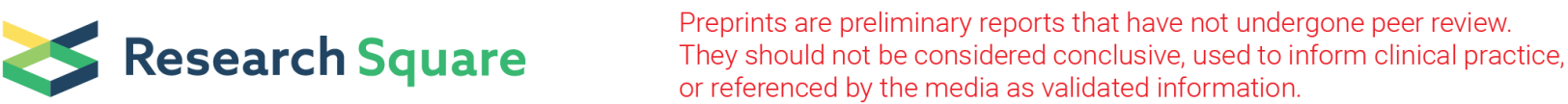

\section{The Impacts of Implementing Primary Care Indicators and Related Financial Incentives on the Trends of Vaccination Coverage Against Influenza Among Elderly People in Hungary}

Gergő Szőllő̃si

University of Debrecen

Ala'a Al-Tammemi

University of Debrecen

Szilvia Harsányi

University of Debrecen

Szabolcs Dr. Garbóczy

University of Debrecen

Nándor Dr.Balogh

University of Debrecen

Ildikó Dr. Simon

University of Debrecen

László Dr. Kolozsvári ( $\square$ kolozsvari.laszlo@med.unideb.hu )

University of Debrecen

\section{Research Article}

Keywords: Influenza, vaccination coverage, indicators, financial incentive, primary care, Hungary

Posted Date: December 31st, 2020

DOI: https://doi.org/10.21203/rs.3.rs-132452/v1

License: (c) (1) This work is licensed under a Creative Commons Attribution 4.0 International License. Read Full License 


\section{Abstract}

Background Influenza is considered one of the most important global public health issues, and a main contributing factor to significant mortality and morbidity across many countries worldwide, especially among elderlies. This study aimed to evaluate the changes of flu vaccination coverage among elderlies in Hungary over the past years and to analyze the effect of implementing financial incentive related primary care indicators on the vaccination coverage.

Methods $95 \%$ Confidence intervals for proportions of people aged 65 or above regarding influenza vaccination status were calculated yearly in order to detect the trends of vaccination coverage in Hungary before and after an 8-year period of the introduction of indicators system.

Results Despite the financial motivational incentives provided to general practitioners to vaccinate their patients against influenza, the vaccination coverage is declining in recent years i.e. before the implementation: $36.01 \%$ (95\% Cl: 35.98 - 36.04); after the implementation: $28.03 \%$ (95\% Cl : 28.01 28.06).

Conclusions According to our results, the implementation of indicators system and related financial incentives could not exploit the potential opportunities in the aspect of increasing the flu vaccination coverage among elderlies in Hungary. Therefore, increasing the flu vaccination coverage should be achieved, not only with free -of- charge vaccines and financial incentives, but also with other possible options such as raising the awareness among people and implementing an effective, follow up system.

\section{Background}

Infectious diseases have recently become the focus of attention. There is also a significant public debate about the different infectious diseases and how to stop them from spreading. One of the most discussed infectious diseases is the influenza and about $30 \%$ of the burden of infectious diseases is related to it. (3) According to the World Health Organization [WHO] influenza affects $5-15 \%$ of the population every year worldwide. This translates into about 1 billion infected individuals per year, with between 3 and 5 million serious infections, resulting deaths between 290.000 and 650.000 most of influenza deaths occur among people aged 65 years or more especially in low- and middle-income countries (1). The European Centre for Disease Prevention and Control [ECDC] estimates that nearly 40.000 people die from influenza in the European Union / European Economic Area [EU / EEA] countries each year (2). The WHO has set a target to vaccinate people who are above the age of 60 , to reach an influenza vaccination coverage of $50 \%$ by the year 2006 and 75\% by the year 2010. In 2011, the Department of Health and Human Services [DHHS] ventured to ask governments to vaccinate $90 \%$ of the elderly by 2020 .

Influenza also has severe economic impact, which can be direct (hospitalization, medication use) and indirect (out of work, loss of productivity). For example, the total economic impact of seasonal influenza in the United States of America was estimated to be $\$ 87.1$ billion in 2003, with a direct medical cost of $\$$ 
10.4 billion US dollars with $64 \%$ of the total burden was attributed to influenza among patients aged 65 or more (5).

The influenza viruses are usually co-circulating with different respiratory pathogens and the diagnosis is often based on clinical signs and symptoms, that is why the estimation of the burden of influenza is difficult, because it changes from year to year, (4). Immunization can effectively reduce the prevalence and burdens of the influenza, but cost-effectiveness is influenced by many factors, for example the efficiency and strain of vaccine, the severity of the flu cases, etc. (4)

The risk of mortality from influenza is sharply rising after age 65 . There is no standardized definition of what exactly "elderly" means regarding the target group of vaccination. It could vary from country to country between 50 and 65 years. Nonetheless, vaccination of the elderly is an important key to almost all flu strategies. The vaccination coverage rate among the elderly varies in most countries, but the level which was set by the WHO only in a very few cases has been achieved (6). Concerning vaccine safety, the elderly people are generally well-tolerating the flu vaccine, thus the administration is considered safe for them. Clinically significant or serious side effects are rare; nevertheless, after vaccination 30 to $40 \%$ of the elderly develop protection against influenza (7). Vaccination against influenza is recommended every year to achieve adequate protection. Unlike the long-term immunity following natural infection, the protection afforded by the flu vaccine is relatively short, especially in the elderly (10). In 2006 a large systematic review found that the flu vaccination of the elderly population can be cost-effective or costsaving (11).

\section{The family physicians' indicator-based performance evaluation system in Hungary}

The Hungarian Ministry of Health commenced the implementation of the indicator-based performance evaluation system in 2009 in order to stimulate the improvement of the quality of primary care based on international experiences (for eg. Organisation for Economic Co-operation and Development [OECD], Quality and Outcomes Framework [QOF]) (12).

A long-term goal was to make every OECD health indicator available for Hungarian health care providers. The National Health Insurance Fund of Hungary is supervising this monitoring-financing system. The incentives paid due the indicator system is 608.3 million Hungarian Forint [HUF] which is equal to 1.73 million Euros (EUR) per month in Hungary. The calculation and evaluation of the indicators are established by county (there are 19 counties in Hungary) and capital level (Budapest) and also calculated according to the practice type (there are adult only $(n=3504)$, paediatric only $(n=1500)$ and mixed practices ( $n=1501)$ in Hungary).

The Hungarian performance-based indicators consist of 16 indicators in adult and mixed practices as well, from which one of the important family physicians' indicators is the flu vaccination coverage among adults aged 65 or above. The influenza vaccine is free for all Hungarian citizens over the age of 60 years 
(paid by the government), which can be requested by the patient from the assigned family physician and the vaccine is administered at the family physicians' clinic. The other monitoring indicators cover the following areas: mammography coverage, treatment of patients with increased blood pressure, blood lipid test among patients with increased blood pressure and diabetic patients, treatment of patients with ischaemic heart disease, treatment of diabetic patients, medications and follow up of patients with chronic obstructive pulmonary disease [COPD], rate of referrals, antibiotic and pharmaceutical prescription indicators (12).

The relevant indicator - influenza vaccination rate among elderlies - which we have investigated in our study demonstrates the proportion of insured persons aged 65 or above who have received a flu vaccination within the last 12 months. These indicators are calculated on a yearly basis. Every insured person is counted only once within the 1-year period. This indicator is only calculated in the adult and mixed practices. The higher the vaccination coverage the better the performance of the practice is.

The main purpose of this indicator is to increase the rate of vaccination among people above 65 years of age. All practices that reach the target value appropriate to their area of care are receiving extra financial incentive (12).

The strengths and the weaknesses of the Hungarian indicator system were highlighted before in other publications (eg. low financial motivation, some indicators cannot be influenced by the GPs), therefore, our present study focuses on the influenza vaccination coverage trends before and after the introduction of the primary care indicator system in Hungary, and whether the financial incentives related indicators can increase the flu vaccination coverage among the elderly or not (13).

Our main aims in the present study were to:

- analyze the impact of implementing the primary care indicators on the trends of influenza vaccination coverage among elderly people in Hungary,

- evaluate the effects and influence of the indicators system on improving the quality of primary care services in the aspect of influenza vaccination,

- compare the overall vaccination coverage proportions before and after the introduction of primary care indicators and the related financial incentives.

\section{Methods}

We used publicly available data (vaccination coverage among people aged 65 and above) regarding the influenza vaccination rate and proportion of elderlies from the homepage of OECD website (14) and data were collected for the period of 2002-2017. During data management we estimated the exact number of persons who were vaccinated using the vaccination coverage rates and the number of Hungarian population aged 65 or above. After that we merged the dataset (2002-2009, 2010-2017) into two exclusive data-groups which contained the overall vaccinated status before and after the implementation of primary care indicators (1st of January 2010). 


\section{Statistical analysis}

In order to establish how the Hungarian vaccination coverage rate among elderlies changed due to the implementation of primary care indicators, $95 \%$ confidence intervals of proportions and the linear equation were calculated and presented in the current study. With this method, we ought to estimate the effect and influence of the chosen primary care indicator on the quality improvement regarding primary care as well.

\section{Results}

\section{Secular trend}

The influenza vaccination coverage was $36.80(95 \% \mathrm{Cl}: 36.72-36.88)$ in 2002 and apart from a few minor increases of the rates, the vaccination significantly decreased to $26.80 \%$ (95\% Cl: $26.74-26.86)$ by the year of 2017. It means that approximately 571105 people received a flu shot in 2002 and this number has declined to 489965 by the year of 2017 corresponding to a 10\% reduction.

According to our results a decreasing trend $(y=-0.009+0.400)$ could be observed during the period of 2002-2017 regarding vaccination of elderlies against influenza. Nevertheless, it seems that the Hungarian free-of-charge influenza vaccination coverage was significantly below the recommended $75 \%$ value established by the WHO (Fig. 1.).

\section{Comparison of the overall proportions before and after the implementation of the financial incentives}

The overall vaccination coverage was $36.01 \%(95 \% \mathrm{Cl}: 35.98 \%-36.04 \%)$ on the merged data for the period 2002-2009. However, the analysis conducted on the following period of 2010-2017 showed that the vaccination rate has significantly decreased to $28.03 \%$ (95\% Cl: $28.01-28.06)$ (Table 1.)

According to our results it seems that the implementation of financing incentive indicators did not increase the overall vaccination coverage, unexpectedly, a significant decreasing trend was observed.

\section{Discussion}

The influenza vaccination coverage is still insufficient despite the introduction of the financial incentive related primary care indicators in Hungary. Although there is a financial motivation for the family physicians to vaccinate their elderly patients against influenza, the flu vaccination coverage is declining in recent years.

However, the results should be interpreted with caution due to several factors which were not investigated this current study (e.g. individual behavioral factors, attitude towards the family physicians). According to the literature there are several factors which might influence the vaccination. A study in 1989 found that 
older people desire interventions that prolong their lives. Over 65, influenza vaccination is one such intervention. A study of people living in an elderly home found that it was mostly relatives who agreed to such interventions (including interviewing health care providers). The elderly people are often in need of guardianship because of the growing cognitive decline and other health burdens. In the case of patients under guardianship, it may be that the care of the elderly is nowadays solved by professional guardians and it is not the family member who makes the decision in their best interest anymore. It is also important to educate the elderly in a way that is understandable to them so that they can give their informed consent (eg. using vignette with simple language) (15).

According to a study of Mayo AM and Cobler S. in 2004, the best motivating factors for accepting a vaccine were that it had been received previously by the person (93\%) and that it was recommended by a health care professional (62\%). Besides, the biggest barriers were the fear of side effects (35\%) and fear of catching the flu (30\%). The basic idea would be that patients perceive themselves to be at high risk and will therefore do more for their health. But even though patients may know what it means to be high risk, they do not consider themselves to fall into this category. It was found that the vaccinated and non vaccinated people had significant differences regarding to four major categories: age, whether they classified themselves as high-risk patients, the number of years of education, and self-rated health. Vaccinated people were older, more likely to be classified themselves as high-risk patients, have higher educational level, and they considered their health worse (16).

An American study found similar results: older people $(>85)$ and those who had at least high school graduates were more likely to accept the vaccine. The White population was also more likely to receive the vaccine than the Hispanic or African Americans population. Smokers were less likely to receive the vaccination, while those who reported not having seen by a doctor in the past year were $28 \%$ less vaccinated, than those who had seen a doctor within one year. When people who did not ask the vaccine was asked why they did not, the distribution of responses was as follows: $20 \%$ said they were afraid of side effects or that the vaccine could cause disease, $17 \%$ thought that vaccination would not stop the flu or did not know they needed it, $13 \%$ thought the vaccine itself could cause the flu. Only $5 \%$ said they did not ask because they were not recommended by their doctor to do so and $1 \%$ did not ask because of its price (17).

Furthermore, an Italian study found that older people, those who spent less time in education and less healthy patients were more likely to be vaccinated. The latter group includes people with chronic illnesses who have declared themselves to be in poor health. They also found that higher household income and smoking reduced the likelihood of someone being vaccinated (18).

In the US, in the 2018-2019 season, $68.1 \%$ of those over 65 were vaccinated (19). According to the ECDC report, 19 of the EU / EEA Member States have reported coverage data of older age groups in recent years. None of these countries achieved the target coverage of $75 \%$. While in the previous study 5 countries were unable to report vaccine coverage, this was 11 in the latest survey. Coverage has declined or stagnated in several countries (20). 
There is a significant number of sites on the Internet that are against vaccination. The phenomenon known as Web 2.0 has become ubiquitous and means that interactive and user-generated content is shared. At the same time, a new paradigm was emerging that placed power in the hands of patients instead of the doctors, questioning the legitimacy of science. Together, they have created an environment in which anti-vaccine movements can effectively communicate their messages. People often turn to the Internet for advice on vaccination, which greatly influences them in their decision-making. There have been frequent claims on the Internet that the vaccine causes illness; ineffective; parts of a governmental / health / pharmaceutical conspiracy; the main pharmaceutical trend is incorrect and corrupt (21). The impact of this movement is illustrated by an online survey conducted during a flu pandemic in Canada, showing that $23.4 \%$ of people thought the vaccine was safe, $41.4 \%$ considered it unsafe and $35.2 \%$ were ambivalent about its safety (22). Looking further into this area, during H1N1 influenza in 2009-2010, there was a great deal of fear of vaccines due to the promotion of anti-vaccine activists. In the US, 70 million doses were destroyed, although there was no evidence that the vaccines did harm (23).

\section{Conclusions}

Our study clearly demonstrated that the financial incentives provided by the Hungarian Ministry of Health to family physicians are not sufficient to increase the rates of influenza vaccination in people over 65 years of age. Vaccination coverage has not increased, but rather has decreased significantly over the years. It can be concluded that increasing the motivation of physicians alone is not expected to increase vaccination rates. Additional factors discussed in the previous section may also be important in increasing the success of this campaign. We would like to provide a guidance for the Hungarian and foreign decision makers and alternatives on how it is possible to retard the decrease in vaccination coverage, and how we can approach the target set by the WHO. It is very important to increase the flu vaccination coverage to lower the number of the flu-like cases, not only with free of charge vaccines and financial incentives, but with other possible opitons (e.g. raising the awareness, better follow up sytem) in order to improve the life quality of the patients and to decrease the burden of the disease.

\section{List Of Abbreviations}

AIDS - acquired immunodeficiency syndrome

COPD - Chronic Obstructive Pulmonary Disease

DHHS - Department of Health and Human Services

ECDC - European Centre for Disease Prevention and Control

EEA - European Economic Area

EU - European Union 
HIV - human immunodeficiency viruses

HUF - Hungarian Forint

OECD - Organisation for Economic Co-operation and Development

QOF - Quality and Outcomes Framework

US - United States

WHO - World Health Organization

\section{Declarations}

\section{Ethics approval and consent to participate}

Not applicable. We did not use individual data of human or animal studies.

\section{Consent for publication}

Not applicable.

\section{Availability of data and materials}

The datasets analyzed during the current study are available from the from the corresponding author on reasonable request and from publicly available websites:

- National Health Insurance Fund of Hungary. The primary care indicator system.

http://www.neak.gov.hu/virtualis_rovat/altfin_virt_dok2/Alapellatas/hsz_indikator. Accessed on 17 Dec 2020.

- Influenza vaccination rates (indicator). https://data.oecd.org/healthcare/influenza-vaccinationrates.htm. Accessed on 17 December 2020.

- European Centre for Disease Prevention and Control. Seasonal influenza vaccination and antiviral use in EU/EEA Member States. https://www.ecdc.europa.eu/en/publications-data/seasonal-influenzavaccination-antiviral-use-eu-eea-member-states (2018). Accessed on 17 December 2020.

\section{Competing interests}

The authors declare that they have no competing interests.

\section{Funding}

The authors received no financial support for the research, authorship, and/or publication of this article. 


\section{Authors' contributions}

All authors worked on the study design, text writing and editing of the manuscript (GJS, ABA, SH, SG, NB, IS, LRK). GJS and LRK done the data management, analysis, and extraction. The figure and the table were made by SZH, GJS, LRK. The literature search was done by ABA, SH, SG, GJS, LRK. The drafting and the interpretation of the of the manuscript was made in close collaboration by all co-authors. All authors read and approved the final manuscript (GJS, ABA, SH, SG, NB, IS, LRK).

\section{Acknowledgements}

Not applicable.

\section{References}

1. A Danielle luliano, Katherine M Roguski, Howard H Chang, David J Muscatello, Rakhee Palekar, Stefano Tempia, et al. Estimates of global seasonal influenza-associated respiratory mortality: a modelling study. Lancet. 2018;391(10127):1285-1300.

2. A Nicoll, B C Ciancio, V Lopez Chavarrias, K Mølbak, R Pebody, B Pedzinski, et al. Influenza-related deaths - Available methods for estimating numbers and detecting patterns for seasonal and pandemic influenza in Europe. Euro Surveill. 2012;17(18):20162.

3. Alessandro Cassini, Edoardo Colzani, Alessandro Pini, Marie-Josee J Mangen, Dietrich Plass, Scott A McDonald, et al. Impact of infectious diseases on population health using incidence-based disabilityadjusted life years (DALYs): Results from the burden of communicable diseases in Europe study, European Union and European economic countries, 2009 to 2013. Euro Surveill. 2018;23(16):1700454.

4. Jan Smetana, Roman Chlibek, Jana Shaw, Miroslav Splino, Roman Prymula. Influenza vaccination in the elderly. Hum Vaccin and Immunother. 2018;14(3):540-549.

5. Noelle-Angelique M Molinari, Ismael R Ortega-Sanchez, Mark L Messonnier, William W Thompson, Pascale M Wortley, Eric Weintraub, et al. The annual impact of seasonal influenza in the US: Measuring disease burden and costs. Vaccine. 2007;25(27):5086-96.

6. Abraham Palache, Valerie Oriol-Mathieu, Mireli Fino, Margarita Xydia-Charmanta, Influenza Vaccine Supply task force (IFPMA IVS). Seasonal influenza vaccine dose distribution in 195 countries (20042013): Little progress in estimated global vaccination coverage. Vaccine. 2015;33(42):5598-5605.

7. Katherine Goodwin, Cécile Viboud, Lone Simonsen. Antibody response to influenza vaccination in the elderly: A quantitative review. Vaccine. 2006;24(8):1159-69.

8. Luzhao Feng, David K Shay, Yong Jiang, Hong Zhou, Xin Chen, Yingdong Zheng, et al. Influenzaassociated mortality in temperate and subtropical Chinese cities, 2003-2008. Bull World Health Organ. 2012;90(4):279-288B.

9. Anthony E Fiore, Timothy M Uyeki, Karen Broder, Lyn Finelli, Gary L Euler, James A Singleton, et al. Prevention and control of influenza with vaccines: Recommendations of the Advisory Committee on 
Immunization Practices (ACIP), 2010. MMWR Recomm Rep. 2010;59(RR-8):1-62.

10. Pritish K Tosh, Robert M Jacobson, Gregory A Poland. Influenza vaccines: From surveillance through production to protection. Mayo Clin Proc. 2010;85(3):257 - 73.

11. Maarten J Postma, Rob Pm Baltussen, Abraham M Palache, Jan C Wilschut. Further evidence for favorable cost-effectiveness of elderly influenza vaccination. Expert Rev Pharmacoecon Outcomes Res. 2006;6(2):215-27.

12. The primary care indicator system. National Health Insurance Fund of Hungary. http://www.neak.gov.hu/virtualis_rovat/altfin_virt_dok2/Alapellatas/hsz_indikator. Accessed on 17 Dec 2020.

13. László Róbert Kolozsvári, Imre Rurik. [Quality evaluation of primary care service performance. What are the problems with the recent Hungarian primary care indicators]. Orv Hetil. 2016;157(9):328-35.

14. Influenza vaccination rates (indicator). OECD. 2020. https://data.oecd.org/healthcare/influenzavaccination-rates.htm. Accessed on 17 December 2020.

15. J G Ouslander, A J Tymchuk, B Rahbar. Health care decisions among elderly long-term care residents and their potential proxies. Arch Intern Med. 1989;149(6):1367-72.

16. Ann M Mayo, Steffanie Cobler. Flu vaccines and patient decision making: what we need to know. J Am Acad Nurse Pract. 2004;16(9):402-10.

17. Kimberly A Lochner, Marc Wynne. Flu shots and the characteristics of unvaccinated elderly Medicare beneficiaries. Medicare Medicaid Res Rev. 2011;1(4):001.04.b01.

18. Carlos Chiatti, Pamela Barbadoro, Giovanni Lamura, Lucia Pennacchietti, Francesco Di Stanislao, Marcello M D'Errico, et al. Influenza vaccine uptake among community-dwelling Italian elderly: Results from a large cross-sectional study. BMC Public Health. 2011;11:207.

19. Centers for Disease Control and Prevention: Flu Vaccination Coverage, United States, 2018-19 Influenza Season. https://www.cdc.gov/flu/fluvaxview/coverage-1819estimates.htm (2019). Accessed on 17 December 2020.

20. European Centre for Disease Prevention and Control. Seasonal influenza vaccination and antiviral use in EU/EEA Member States - Overview of vaccine recommendations for 2017-2018 and vaccination coverage rates for 2015-2016 and 2016-2017 influenza seasons.

https://www.ecdc.europa.eu/en/publications-data/seasonal-influenza-vaccination-antiviral-use-eueea-member-states (2018). Accessed on 17 December 2020.

21. Anna Kata. Anti-vaccine activists, Web 2.0, and the postmodern paradigm - An overview of tactics and tropes used online by the anti-vaccination movement. Vaccine. 2012;30(25):3778-89.

22. Neil Seeman, Alton Ing, Carlos Rizo. Assessing and responding in real time to online anti-vaccine sentiment during a flu pandemic. Healthc Q. 2010;13 Spec No:8-15.

23. Gregory A Poland, Robert M Jacobson. The age-old struggle against the antivaccinationists. New England Journal of Medicine. 2011;364(2):97-9. 


\section{Tables}

Due to technical limitations, table 1 is only available as a download in the Supplemental Files section.

\section{Figures}

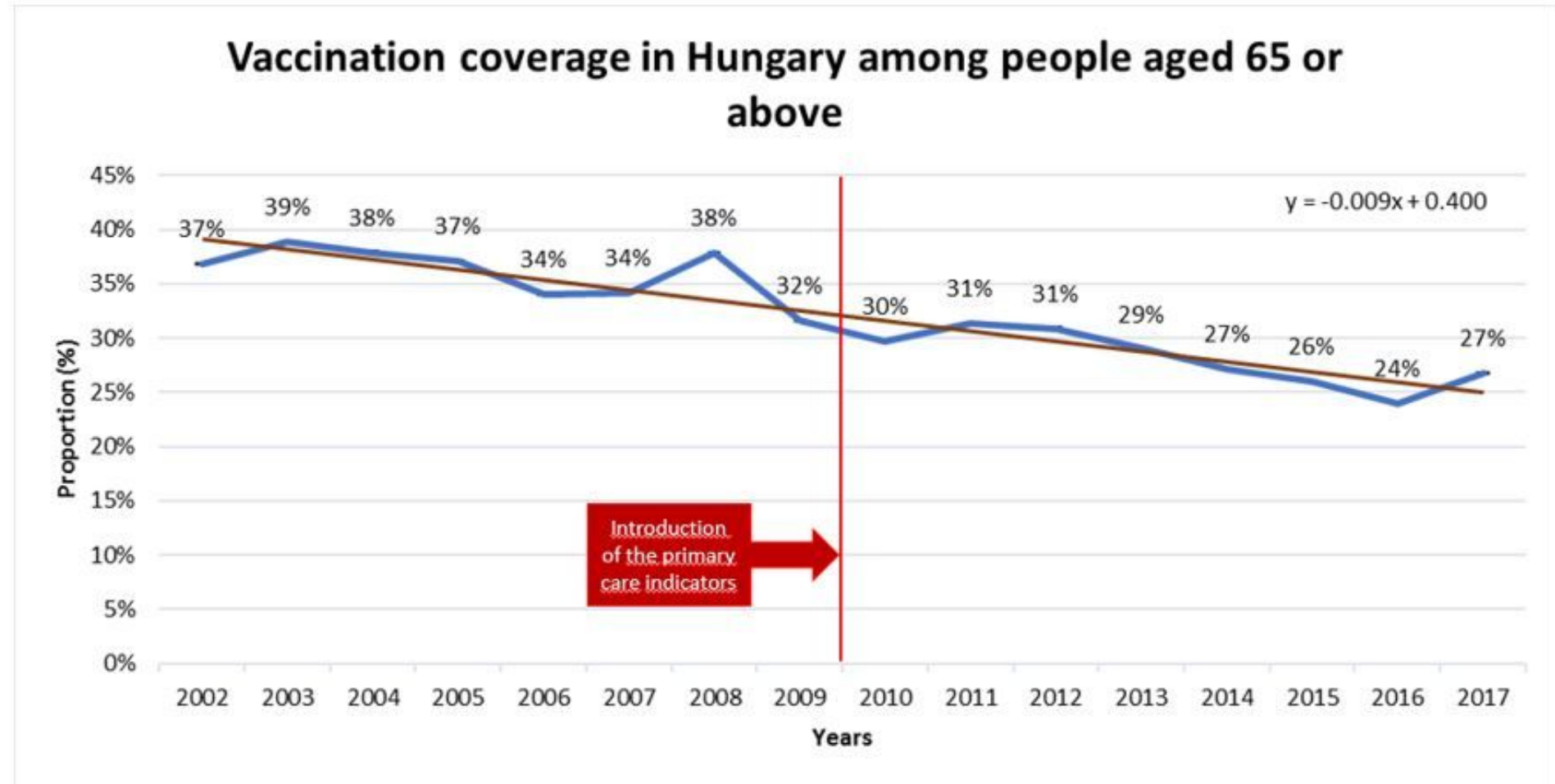

Figure 1

Influenza vaccination coverage among the elderly over 65 years of age in Hungary. A decreasing trend can be seen in the proportion of the vaccinated elderly in Hungary between 2002 and 2017.

\section{Supplementary Files}

This is a list of supplementary files associated with this preprint. Click to download.

- Table1.jpg 